\title{
Prognostic value of stress-only and stress-rest normal gated SPECT imaging: higher incidence of cardiac hard events in diabetic patients who underwent full stress-rest imaging
}

Milan Kamineka , Iva Metelkovaa ${ }^{a}$ Miroslava Budikova ${ }^{a}$, Lenka Henzlova ${ }^{a}$, Pavel Korandaa ${ }^{a}$ Vladimir Kincl ${ }^{b}$, Adela Drozdova $^{b}$

Background. The European procedural guidelines for cardiac gated SPECT imaging demonstrate considerable variability in recommended administered radiopharmaceutical activity and imaging protocols. This study compared stress-only and stress-rest protocols to evaluate the safety of stress-only imaging, and to identify characteristics of patients who need full stress-rest imaging.

Methods. Patients referred for a chest pain were scheduled for stress-rest gated SPECT imaging. If the stress images were interpreted as normal according to the perfusion and left ventricular function, the examination of patients was finished and patients did not undergo the rest imaging. A total number of 1063 patients was included (mean age $61 \pm$ 11 years). These patients have been followed for hard cardiac events, i.e. cardiac deaths or nonfatal myocardial infarction. Results. During a follow-up of $3.2 \pm 2.5$ years, hard events occurred in 12 patients with normal SPECT and 59 with abnormal SPECT had hard events $(0.7 \mathrm{vs.} 3.6 \%$ /year, $P<0.001)$. Among the 536 patients with normal study, there was no significantly lower incidence of hard events in the subgroup of patients with stress-only imaging $(0.6 \mathrm{vs} .0 .8 \%$ /year, $P=0.641$ ). Diabetes mellitus was an independent predictor of hard events in patients with normal SPECT ( $1.3 \mathrm{vs.} 0.5 \% /$ year, $P<0.001)$. We found a higher incidence of hard events in diabetic patients with normal study with the necessity of full stress-rest imaging in comparison with those with stress-only imaging ( $1.7 \mathrm{vs.} 0.7 \% /$ year, $P<0.001)$.

Conclusions. Our results support the good prognosis of normal stress-only study. Diabetes mellitus was an independent predictor of hard events in patients with normal SPECT. Diabetic patients with normal results who required additional rest imaging had significant adverse outcome.

Key words: myocardial perfusion imaging, coronary artery disease, prognosis, stress-only imaging, diabetes mellitus

Received: December 30, 2013; Accepted with revision: May 7, 2014; Available online: May 30, 2014 http://dx.doi.org/10.5507/bp.2014.024

\footnotetext{
${ }^{a}$ Department of Nuclear Medicine, Faculty of Medicine and Dentistry, Palacky University Olomouc and University Hospital Olomouc, Czech Republic.

'International Clinical Research Center, Centrum of Molecular Imaging, Brno

Corresponding author: Milan Kaminek, e-mail:milan.kaminek@fnol.cz
}

\section{INTRODUCTION}

Gated single-photon emission tomography (SPECT) is a well-established method for myocardial perfusion, function, and viability imaging in patients with suspected or known coronary artery disease (CAD) $\left(\right.$ ref $\left.^{1-3}\right)$. 15-20 million cardiac SPECT imaging are performed annually worldwide. The EANM/ESC procedural guidelines for cardiac gated SPECT imaging demonstrate considerable variability in recommended administered radiopharmaceutical activity and imaging protocols ${ }^{3}$. In a majority of European countries, all patients still receive both stress and rest tracer activity using a dual day stress-rest imaging ${ }^{4,5}$. However, there is an opportunity to perform stressonly imaging in those patients with normal stress pattern. This approach leads to significantly reduced radiation burden, time, and cost from myocardial perfusion imaging. In our laboratory, about one half of the population referred for chest pain undergoes stress-only imaging because of normal stress perfusion and left ventricular gated SPECT pattern.
However, there is a well known limitation of SPECT: the evaluation of relative perfusion only. The defect visualized after a stress test is related to the most severe coronary artery stenosis but may not fully reflect the extent of CAD. Moreover, in a few patients with balanced multivessel CAD, perfusion defects may be not evident. The use of other indirect hemodynamic indices of extensive CAD, such as worsening of the left ventricular ejection fraction (EF) or wall motion, increased tracer lung uptake or transient ischemic dilatation (TID) of the left ventricle, have been validated in order to improve the assessment of prognosis ${ }^{6-10}$. Some patients with an absence of perfusion abnormality can have benefit from a complete examination to compare stress and rest left ventricular function. However, it is not easy to identify those patients.

The purpose of this study was to compare stress-only and stress-rest protocols to evaluate the safety of stress-only imaging, and to identify the characteristics of patients who need full stress-rest imaging. 
Table 1. Baseline clinical characteristics of patients with normal SPECT according to imaging protocol.

\begin{tabular}{|c|c|c|c|c|}
\hline & $\begin{array}{c}\text { Global } \\
(n=536)\end{array}$ & $\begin{array}{l}\text { Stress only } \\
(\mathrm{n}=259)\end{array}$ & $\begin{array}{l}\text { Stress-rest } \\
(\mathrm{n}=277)\end{array}$ & $P$ \\
\hline Age (years), mean \pm SD & $59 \pm 11$ & $59 \pm 9$ & $58 \pm 13$ & 0.508 \\
\hline Male, n (\%) & $265(49 \%)$ & $99(38 \%)$ & $165(59 \%)$ & $<0.001$ \\
\hline $\begin{array}{l}\text { Gated SPECT variables, mean } \pm \mathrm{S} \\
\text { Summed stress score } \\
\text { Ejection fraction }(\%) \\
\text { End-diastolic volume }(\mathrm{ml}) \\
\text { End-systolic Volume }(\mathrm{ml})\end{array}$ & $\begin{array}{c}1.1 \pm 2.8 \\
64 \pm 10 \\
104 \pm 44 \\
41 \pm 32\end{array}$ & $\begin{array}{c}0.5 \pm 2.2 \\
69 \pm 8 \\
85 \pm 24 \\
27 \pm 12\end{array}$ & $\begin{array}{c}1.7 \pm 3.2 \\
60 \pm 11 \\
120 \pm 50 \\
53 \pm 38\end{array}$ & $\begin{array}{l}<0.001 \\
<0.001 \\
<0.001 \\
<0.001\end{array}$ \\
\hline Diabetes mellitus, n (\%) & $123(23 \%)$ & $47(18 \%)$ & $76(27 \%)$ & 0.011 \\
\hline Left bundle branch block, n (\%) & $21(3.9 \%)$ & $1(0.4 \%)$ & $20(7.2 \%)$ & $<0.001$ \\
\hline Dilated cardiomyopathy, n (\%) & $24(4.5 \%)$ & $0(0 \%)$ & $24(8.7 \%)$ & $<0.001$ \\
\hline End stage of renal failure, $\mathrm{n}(\%)$ & $26(4.9 \%)$ & $1(0.4 \%)$ & $25(9 \%)$ & $<0.001$ \\
\hline Previous revascularization, $\mathrm{n}(\%)$ & $85(15.9 \%)$ & $19(7.3 \%)$ & $66(23.8 \%)$ & $<0.001$ \\
\hline
\end{tabular}

\section{PATIENTS AND METHODS}

\section{Study population and follow-up}

Patients referred for cardiac gated SPECT imaging were followed (mean $3.2 \pm 2.5$ years) for the occurrence of hard events: cardiac death or nonfatal myocardial infarction (MI). Telephone interviews of patient's physicians and hospital charts review were performed. Follow-up was obtained in 1063 (99\%) patients: 680 males and 383 females, mean age $61 \pm 11$ years, 166 patients after previous MI, 222 after coronary revascularization, 328 with diabetes mellitus (DM).

\section{Stress testing}

Patients either underwent an exercise test or received intravenous dipyridamole if unable to exercise. The exercise test was performed upright on a bicycle ergometer. Each patient exercised up to $85 \%$ of the age-predicted maximal heart rate or until the onset of angina pectoris, dyspnea or fatigue, dizziness, frequent (>10 per min) multifocal or paired ventricular extrasystoles, ST segment depression $(>0.2 \mathrm{mV})$, or a decrease in blood pressure of $10 \mathrm{~mm} \mathrm{Hg}$ below the peak value of the previous stage. Dipyridamole was administered intravenously at a standard dose of $0.56 \mathrm{mg}$ per $\mathrm{kg}$ of body weight during a 4-min period in the combination with low level of exercise in patients who could not achieve maximal predicted heart rate. Dipyridamole stress only was used for patients with left bundle branch block.

\section{Gated cardiac SPECT}

Stress was done first with Tc-99m compounds (sestamibi or tetrofosmin). If the stress images were interpreted as completely normal according to the perfusion and left ventricular function, the patients were finished and underwent no rest imaging. The patients with abnormal or inconclusive stress images underwent rest study (some had a final normal result according to the perfusion and an attenuation artefact or mild abnormality of the left ventricular function were common findings in these patients). The first dose of tracer was $300 \mathrm{MBq}$ and the second dose was $750 \mathrm{MBq}$ (one-day protocol) or $300 \mathrm{MBq}$ (two-day protocol).

SPECT was performed using a Siemens e.cam camera equipped with a $90^{\circ}$ angled dual head, low-energy highresolution collimators. Images were gated at 8 frames per cardiac cycle. No attenuation correction was applied. We used additional prone position imaging in the case of inferior wall defect (probably caused by attenuation artefact). The summed stress and difference score (SSS, SDS) of myocardial perfusion, the left ventricular EF and end-diastolic/end-systolic volumes (EDV/ESV) were calculated automatically using the 4D-MSPECT (The University of Michigan, Ann Arbor, MI, USA) software.

\section{Statistical Analysis}

All continuous data are expressed as means (SD, standard deviation) and noncontinuous variables are expressed as percentages. Continuous variables were compared using nonparametric Mann-Whitney test because the normality of data was violated. Differences between proportions were compared using the $\chi^{2}$ test. Probability values $<0.05$ were considered significant.

\section{RESULTS}

There was a significantly lower incidence of hard events in patients with normal study (no cardiac death and 12 nonfatal $\mathrm{MI}$ ) in comparison with those with abnormal SPECT (17 cardiac deaths and 42 nonfatal MI, $P$ $<0.001)$. Further analysis was performed in the subgroup of 536 patients with normal study. There was no significantly lower incidence of hard events in 259 patients with stress-only imaging in comparison with those 277 patients who underwent full stress-rest imaging ( $P=0.641$, Fig. 1$)$. 


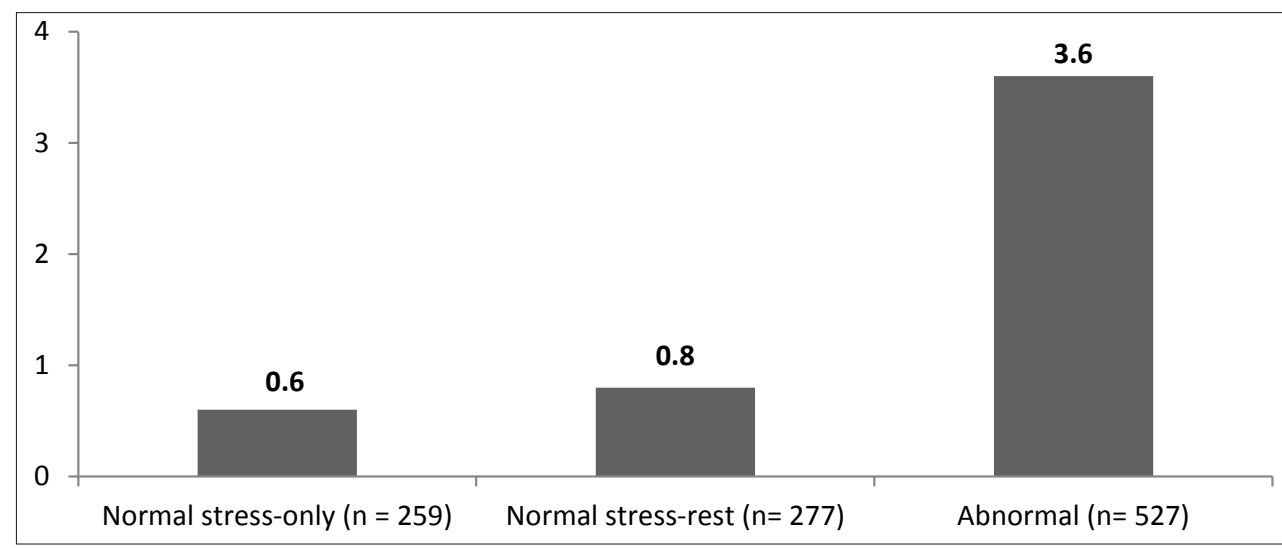

Fig. 1. Frequency of hard events per year (\%) as a function of stress SPECT result.

Significant differences $(P<0.001)$ are present between patients with normal and abnormal study. Patients with normal study are at low risk of cardiac death or nonfatal MI. Not significantly lower incidence of hard events was observed in the subgroup of patients with stress-only imaging $(P=0.641)$

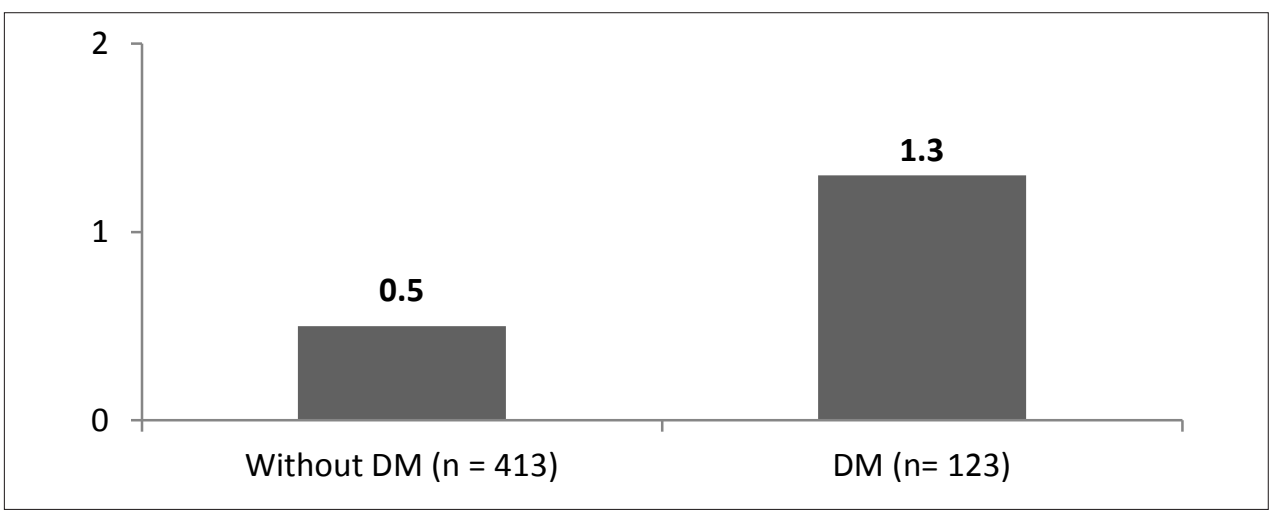

Fig. 2. Hard events per year (\%) in patients with normal study.

Significant higher incidence of hard events was observed in patients with DM $(P<0.001)$.

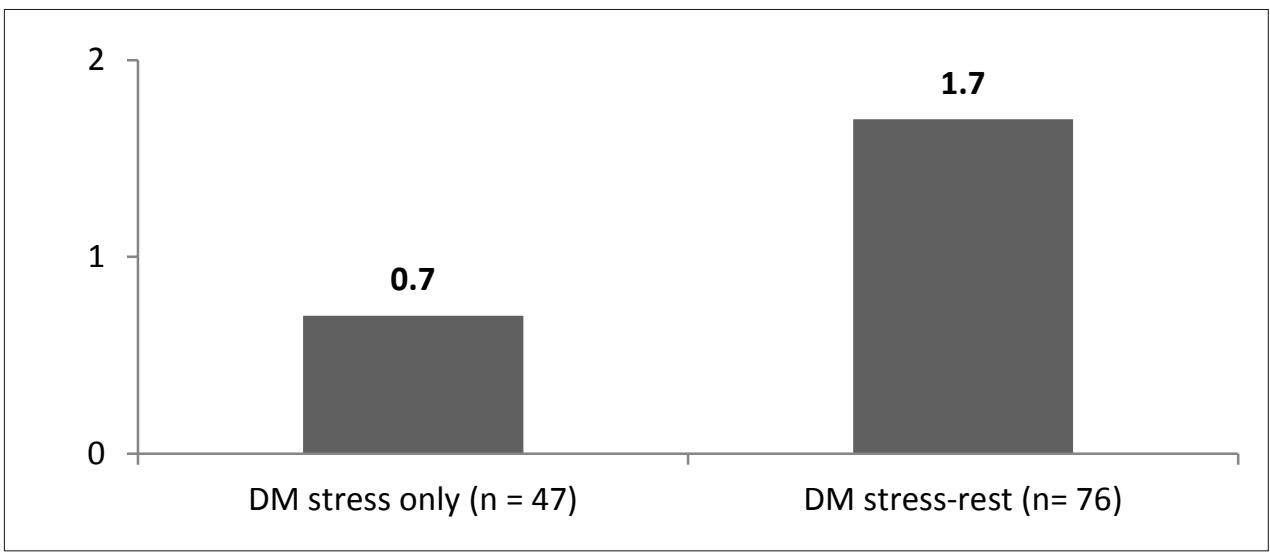

Fig. 3. Hard events per year (\%) in diabetic patients (DM) with normal study.

Significant higher incidence of hard events was observed in patients who underwent full stress-rest imaging $(P<0.001)$. 
Baseline clinical characteristics of patients with normal SPECT according to imaging protocol are summarized in Table 1. Male gender, higher left ventricular volumes and lower EF, DM, left bundle branch block (LBBB), dilated cardiomyopathy, end stage renal failure, and previous revascularization were associated with the implementation of full stress-rest imaging. Diabetes was an independent predictor of hard events in patients with normal SPECT $(P<0.001$, Fig. 2$)$. Among the diabetic patients with normal study, we observed significantly higher incidence of hard events in those individuals who underwent full stress-rest imaging $(P<0.001$, Fig. 3).

\section{DISCUSSION}

Performing stress imaging first should obviate the need for rest imaging in patients with normal left ventricular perfusion and function on stress. A significant reduction in radiation exposure can be achieved with such an approach. Chang et al. ${ }^{11}$ reported stress-only protocol in 8,034 of 16,854 consecutive patients (48\%). In our laboratory, we performed stress-only imaging in $52 \%$ of patients with low to medium probability for CAD in the last five years (31\% of all tested patients including those with known CAD).

A good prognostic value of normal stress-only imaging has been documented by several studies ${ }^{11-13}$. During a mean follow-up of $22.3 \pm 6.4$ months, Gibson et al. ${ }^{12}$ observed a low overall cardiac event rate at $0.6 \%$ in 652 patients with low to medium probability for CAD. In our study, we observed lower annual cardiac hard event rates in patients who underwent stress-only imaging in comparison with those who required additional rest imaging but the results were not significant $(0.6 \%$ vs. $0.8 \%, P=$ $0.641)$. The same result were reported by Shaw LJ et al. $(0.67 \%$ vs. $0.93 \%, P=0.29)\left(\right.$ ref. $\left.^{13}\right)$. This phenomenon is interesting since the stress-only imaging can potentially underestimate balanced multivessel CAD because of the lack of data on rest perfusion and function (TID ratio, worsening of left ventricular EF, etc.).

Therefore, we analyzed patients with and without the necessity for full stress-rest protocol. We found no influence of age between both protocols. In contrast, there was an influence of sex. Men were more likely to need stress-rest imaging due to inferior wall defects caused by high diaphragms. As we expected, LBBB, dilated cardiomyopathy, and end stage renal failure were associated with the necessity for full stress-rest imaging. Stress only imaging was undergone by those with fully normal stress perfusion and left ventricular function. Only 19 out of 85 patients with previous revascularization were completed just after the stress SPECT.

It is well known that DM is associated with accelerated atherothrombosis. An adverse outcome of diabetic patients with normal gated SPECT has been described ${ }^{14}$. In our study, DM was an independent predictor of hard events in patients with normal SPECT ( 1.3 vs. $0.5 \%$ per year, $P<0.001)$. The presence of DM was associated with higher frequency of full stress-rest imaging, probably due to inconclusive stress perfusion images or higher left ventricular volumes $(P=0.011)$. Among diabetic patients with normal study, we observed significantly higher incidence of hard events in those individuals who underwent full stress-rest imaging $(P<0.001)$.

Further risk stratification in diabetic patients is enabled by the combined use of cardiac gated SPECT and coronary artery calcium (CAC) scoring ${ }^{15-16}$. The findings of highly elevated CAC score as well as the post-stress left ventricular stunning allow further risk stratification in patients with or without reversible perfusion abnormalities. In some patients with fully normal SPECT imaging, only an extensive calcium score over 1,000 can predict severe CAD (ref. ${ }^{18}$ ). In diabetic patients, therefore, we recommend the use of a combination of gated SPECT imaging and CAC scoring first, and to continue with rest imaging in all patients with abnormal or inconclusive post-stress gated SPECT findings.

\section{CONCLUSIONS}

Our results support a good prognosis for patients with a normal stress-only study. Diabetes mellitus was an independent predictor of hard events in patients with normal SPECT. In the general population with a normal scan result, there was a lower incidence of hard events in those with stress-only imaging in comparison with those who underwent full stress-rest imaging but the results were not significant. In diabetic patients, however, it was necessary to perform significantly more rest studies. Moreover, we observed a significant adverse outcome in those diabetic patients who required an additional rest imaging (usually due to inconclusive stress perfusion images or relative higher left ventricular volumes) in comparison with those with fully normal stress-only imaging.

\section{ACKNOWLEDGEMENT}

Supported by European Regional Development Fund - Project FNUSA-ICRC (No. CZ.1.05/1.1.00/02.0123).

Author contributions: IM, MB, LH: data collection and data interpretation; PK: study design and data analysis; VK, AD: statistical analysis and manuscript revision.

Conflict of interest statement: The authors declare that there are no conflicts of interest regarding the publication of this article.

\section{REFERENCES}

1. Hachamovitch R, Rozanski A, Shaw LJ, Stone GW, Thomson LE, Friedman JD, Hayes SW, Cohen I, Germano G, Berman DS. Impact of ischaemia and scar on the therapeutic benefit derived from myocardial revascularization vs. medical therapy among patients undergoing stress-rest myocardial perfusion scintigraphy. Eur Heart J 2011;32:1012-24. 
2. Marcassa C, Bax JJ, Bengel F, Hesse B, Petersen CL, Reyes $E$, Underwood R. Clinical value, cost-effectiveness, and safety of myocardial perfusion scintigraphy: a position statement. Eur Heart J 2008;29:557-63.

3. Kamínek $M$, Mysliveček $M$, Škvařilová $M$, Hušák $V$, Koranda $P$ Metelková I, Lang O. Increased prognostic value of combined myocardial perfusion SPECT imaging and the quantification of lung TI201 uptake. Clin Nucl Med 2002;27(4):255-60.

4. Hesse B, Tägil K, Cuocolo A, Anagnostopoulos C, Bardiés M, Bax J, Bengel F, Busemann Sokole E, Davies G, Dondi M, Edenbrandt L, Franken P, Kjaer A, Knuuti J, Lassmann M, Ljungberg M, Marcassa C, Marie PY, McKiddie F, O'Connor M, Prvulovich E, Underwood R, van Eck-Smit B; EANM/ESC Group. EANM/ESC procedural guidelines for myocardial perfusion imaging in nuclear cardiology. Eur J Nucl Med Mol Imaging 2005;32:855-97.

5. Marcassa C, Zoccarato O, Calza P, Campini R. Temporal evolution of administered aktivity in cardiac gated-SPECT and patients' effective dose. Analysis of a historical series. Eur J Nucl Med Mol Imaging 2013;40(3):325-30.

6. Berman DS, Kang X, Slomka PJ, Gerlach J, de Yang L, Hayes SW Friedman JD, Thomson LE, Germano G. Underestimation of extent of ischemia by gated SPECT myocardial perfusion imaging in patients with left main coronary artery disease. J Nucl Cardiol 2007;14(4):521 8.

7. Heiba SI, Santiago J, Mirzaitehrane M, Jana S, Fuat D, Abdel-Dayem HM. Transient postischemic stunning evaluation by stress gated TI201 SPECT myocardial imaging: effect on systolic left ventricular function. J Nucl Cardiol 2002;9:482-92.

8. Sharir T, Bacher-Stier C, Dhar S, Lewin HC, Miranda R, Friedman JD, Germano G, Berman DS. Identification of severe and extensive coronary artery disease by postexercise regional wall motion abnormalities in Tc-99m sestamibi gated single-photon emission computed tomography. Am J Cardiol 2000;86:1171-5.
9. Dona M, Massi L, Settimo L, Bartolini M, Giannì G, Pupi A, Sciagrà R. Prognostic implications of post-stress ejection fraction decrease detected by gated SPECT in the absence of stress-induced perfusion abnormalities. Eur J Nucl Med Mol Imaging 2011;38:485-90.

10. Kamínek $M$, Mysliveček $M$, Škvařilová $M$, Hušák $V$, Koranda $P$ Metelková I, Lang O. Increased prognostic value of combined myocardial perfusion SPECT imaging and the quantification of lung TI201 uptake. Clin Nucl Med 2002;27(4):255-60.

11. Chang SM, Nabi F, Xu J, Raza U, Mahmarian JJ. Normal stress-only versus standard stress/rest myocardial perfusion imaging: similar patient mortality with reduced radiation exposure. J Am Coll Cardiol 2010;55(3):221-30.

12. Gibson PB, Demus D, Noto R, Hudson W, Johnson LL. Low event rate for stress-only perfusion imaging in patients evaluated for chest pain. J Am Coll Cardiol 2002;39(6):999-1004.

13. Shaw LJ, Hage FG, Berman DS, Hachamovitch R, Iskandrian A. Prognosis in the era of comparative effectiveness research: where is nuclear cardiology now and where should it be? J Nucl Cardiol 2012;19(5):1026-43.

14. Romero-Farina G, Candell-Riera J, Aguadé-Bruix S, Cuberas-Borrós G, Ferreira-González I, Nazarena Pizzi M, de León G, Santos A, GarcíaDorado D. Predictive variables for hard cardiac events and coronary revascularization in patiens with normal left ventricular perfusion and systolic function. Eur J Nucl Med Mol Imaging 2013;40:1181-9.

15. Metelková I, Kaminek M, Sovova E, Hutyra M, Budíková M Buriánková E, Formánek R, Henzlová L, Metelka R. Risk stratification by cardiac gated SPECT combined with coronary artery calcium score in patiens with diabetes mellitus and/or renal failure. Vnitr Lek 2010;56(11):1122-9.

16. Ghadri JR, Fiechter M, Fuchs TA, Templin C, Lüscher TF, Kaufmann PA The value of coronary calcium score in daily clinical routine, a case series of patients with extensive coronary calcifications. Int J Cardiol 2013;162(2):e47-49. 